Military Technical College Kobry El-Kobbah, Cairo, Egypt

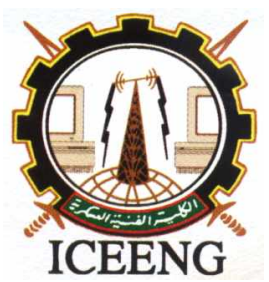

\author{
$6^{\text {th }}$ International Conference \\ on Electrical Engineering \\ ICEENG 2008
}

\title{
Influences of water droplet size and pollution layer permittivity on the electric field and potential distribution on a polluted insulator surface
}

By
M.Y.Kamel**
M.AbouelSaad*
Amged S. El-Wakeel**
H.A.Hassan**

\section{Abstract:}

Presence of water droplets on a polluted outdoor high voltage insulator surface modifies the electric field flux and the equipotential distribution along the insulator surface. The water droplet may appear in different forms and sizes. The form is defined, here, by the angle shape between the water droplet surface and that of the insulator, which is kept constant at 90 degrees. The droplet size is specified by the diameter. The water droplet may measure from less than 90 degrees (hydrophilic) up to greater than 90 degrees (hydrophobic), depending on the properties of insulator surface.

The present work describes a detailed numerical computation of the electric field and the potential distribution in the surroundings of water droplet on a polluted insulator surface. The computations are carried out using the Femlab package 3.1, which is based on the finite element method.

The parameters used in the numerical analysis are the size of the water droplet and the pollution layer permittivity which describes the possible wetting conditions of the layer (i.e. clean, semi-wetted, and fully-wetted polymer surface). Detailed plots of the potential and field distribution in the inter electrode space, with and without the presence of a water droplet, were generated. The results showed that the electric field close to the water droplet tends to be higher as the diameter of the droplet increases, and lower as the relative permittivity of the pollution layer increases.

\section{Keywords:}

electric field, electric potential, water droplet, polluted insulator.

* Professor, Faculty of Engineering, Benha University, Benha, Egypt

** Egyptian Armed Forces 


\section{Introduction:}

In recent years, the application of polymer insulators, to both distribution and transmission lines, has been increasing rapidly because of their advantages of light weight, high mechanical strength, and superior contamination resistant performance[1]. Surface hydrophobicity is one of the important factors that affect the performance of polymer insulators in service. It is usually degraded by environmental conditions such as atmospheric pollution, ultraviolet radiation, acid rain and salt fog. The behavior of the insulator surface when water droplets take place on the surface due to rain and moisture is one of the performance parameters to be determined. Therefore the conductive contamination particles dissolved in the water will discontinue the electric field fluxes[2]. The electric field (E-field) is intensified at the triple junction among the water droplet, air and the insulation material[3]. When erosion and tracking take place due to the existence of local high electric field, it would destroy the hydrophobicity and cause the degradation of the insulator surface [4-6].

Recently, simulation for a still droplet with hemispherical shape has been done to investigate the electric field and potential distributions on the polymer surface[2-5, 7-9]. The present study is focused on establishing a 2D model for calculating the electric field and the potential distribution on the polymer surface with changing droplet size and the pollution layer permittivity under an applied AC voltage by Femlab 3.1 package [10].

\section{Finite Element Simulation Method:}

The Finite Element Method (FEM) was introduced in 1960, and applied in electrical engineering in 1965. Now the FEM is being widely used in electrical engineering as a main numerical calculation method for analytically quantifying and optimizing the performance of an insulator under electro-magnetic fields. FEM is a powerful tool to represent an electric and/or magnetic field over a large, irregularly shaped region[11]. The finite element method for any problem consists of, basically, discretizing the solution domain into a finite number of elements, deriving governing equations for a typical element, assembling of all elements in the solution domain, and finally solving the resulting system of equations[12, 13].

Partial differential equations (PDEs) are discretized by the finite element method to allow users to obtain the solution values, including electric field and potential at any point in the solution domain. The main steps for numerical computation of partial differential equation are as follows:

- Define a PDE problem, by defining the 2-D regions, boundary conditions, and domain parameters,

- Numerically solve the problem, by generating unstructured meshes, discretizing the equations, and producing an approximation to the solution.

- Visualize the results. 
High voltage apparatus, including outdoor insulators, are one of the electrostatic application modes. The 'statics' implies that the time rate of change is slow, and wavelengths are very large compared to the size of the domain of interest[5].

In this case, which is simulated as an electrostatic problem, water droplets and electrodes are assumed to be at a single potential. Therefore, no E-field exists in any conductor and charges have already flow to the outside of the conductor. The electrostatic field simulator solves for the electric potential $V$ in this field equation:

$$
E=-\nabla V
$$

And using one of Maxwell's equations, $\nabla \cdot D=\rho$ and the relationship, $D=\varepsilon E$ one arrives at the Poisson's equation as:

$$
-\nabla \cdot(\varepsilon \nabla \cdot V)=\rho
$$

Where $\varepsilon$ is the permittivity of dielectrics and $\rho$ is the space charge density and $D$ is the electric flux density.

Because there will be an electric field in the dielectric, but no current and no free charges, the relationship that must be satisfied everywhere, including at the interface of the different dielectrics is: $\nabla \cdot D=\rho=0$ or equivalently $\nabla \cdot \varepsilon E=\rho=0$. In other words, the electric field in each material is determined entirely by the permittivity of this dielectric. Applying to Eqn (2) it yields Laplace's equation as:

$\nabla^{2} V=0$

\section{Simulation Configuration:}

The geometry of a polymer insulator sample is represented in two dimensions describing $20 \mathrm{kV}$ phase-to-ground, as shown in Figure 1. The drawing was displayed in a two-dimension plane. On the left of the insulator, the Dirichlet boundary condition prevails, the value of 20000 Volt in this case. It illustrates the HV electrode that is directly in touch to the polymer sample.

At the right part of insulator, there is also Dirichlet boundary condition where its value is zero. This represents the insulator part directly connected to ground. In the display, the Dirichlet boundary condition is indicated in red color.

The other boundary conditions are Neumann conditions, the ones drawn in blue. In this case, the whole values of Neumann boundary condition are zero. In this study, the water droplet is present on the upper part of the polluted insulator sample. One water droplet is drawn to investigate the occurrence of electric potential and electric field distribution changes. 


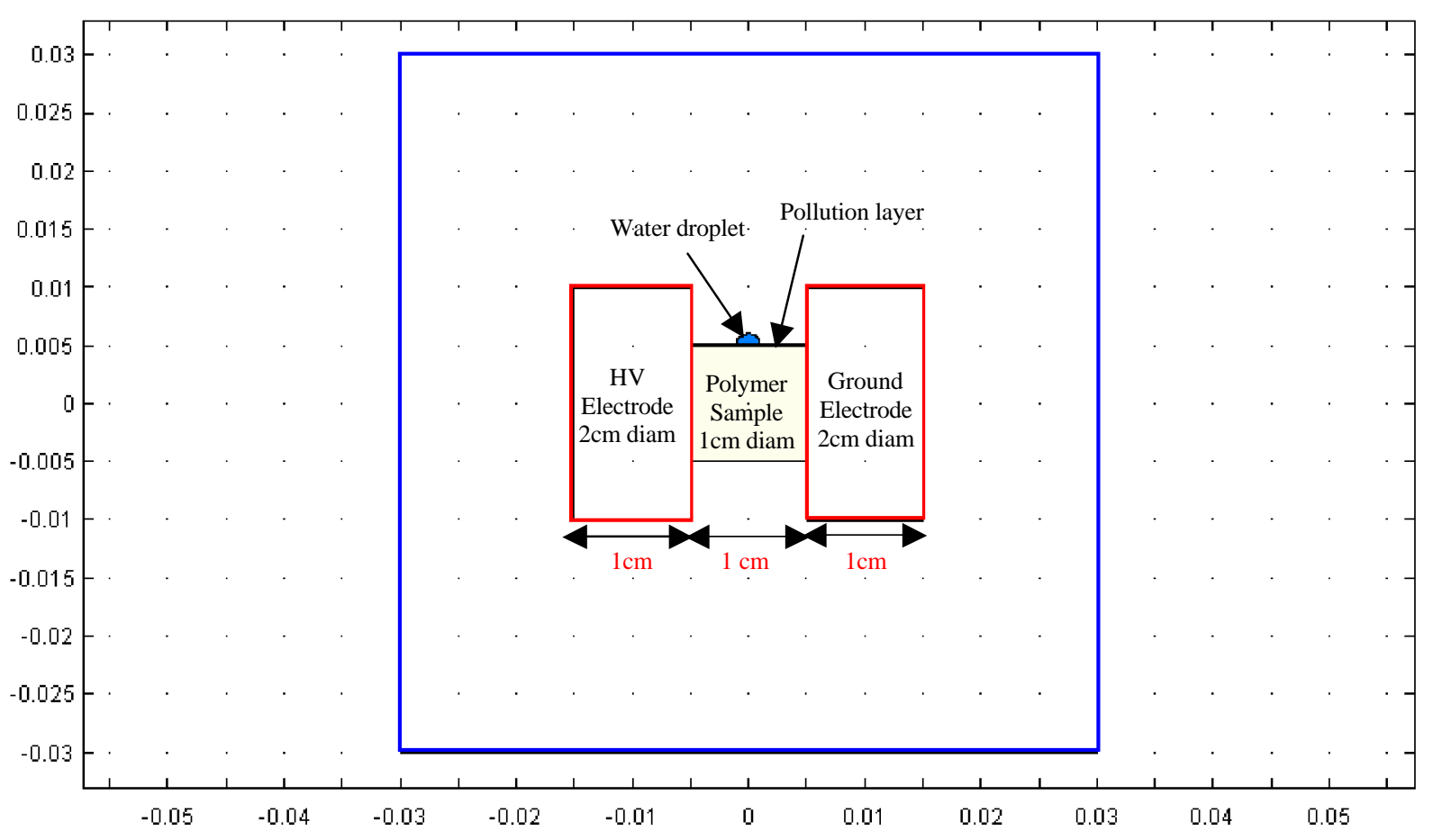

Figure 1: Definition of domain of study, to simulate electric potential and field on an insulator

In this simulation, the dielectric constants of 1, 2.35 and 81, for air, insulation material and water are used respectively. Thus, the permittivity of air, insulator and water were $8.854 \times 10^{-12} \mathrm{~F} / \mathrm{m}, 2.08 \times 10^{-11} \mathrm{~F} / \mathrm{m}$ and $7.172 \times 10^{-10} \mathrm{~F} / \mathrm{m}$ respectively. The dielectric constant of the pollution layer is taking also different values from 2.35 to 81 to simulate different cases of clean, semi-wetted and fully wetted pollution layer respectively.

After inserting the drawing, boundary conditions and the parameter values, the further step is to discretize the domain by construction of mesh. The mesh in triangular elements is shown in Figure 2. The number of elements is carefully chosen to achieve the minimum error. First the simple case of the geometry (only the clean polymer sample) is considered, for which the electric field distribution over the $1 \mathrm{~cm}$ sample is $20 \mathrm{Kv} / \mathrm{cm}$. The mesh was initially created with 970 triangular elements. The electric field in this case was $20.16 \mathrm{Kv} / \mathrm{cm}$ with an error of $(0.8 \%)$. In the next step, 3872 elements were used. The electric field was $20.15 \mathrm{Kv} / \mathrm{cm}$ with an error of $(0.75 \%)$. When the number of elements was increased to 15488 elements, the electric field was found to be $20.14 \mathrm{Kv} / \mathrm{cm}$ with an error of $(0.7 \%)$. It is found that any further increase in the mesh elements does not produce any significant effect on the error. 


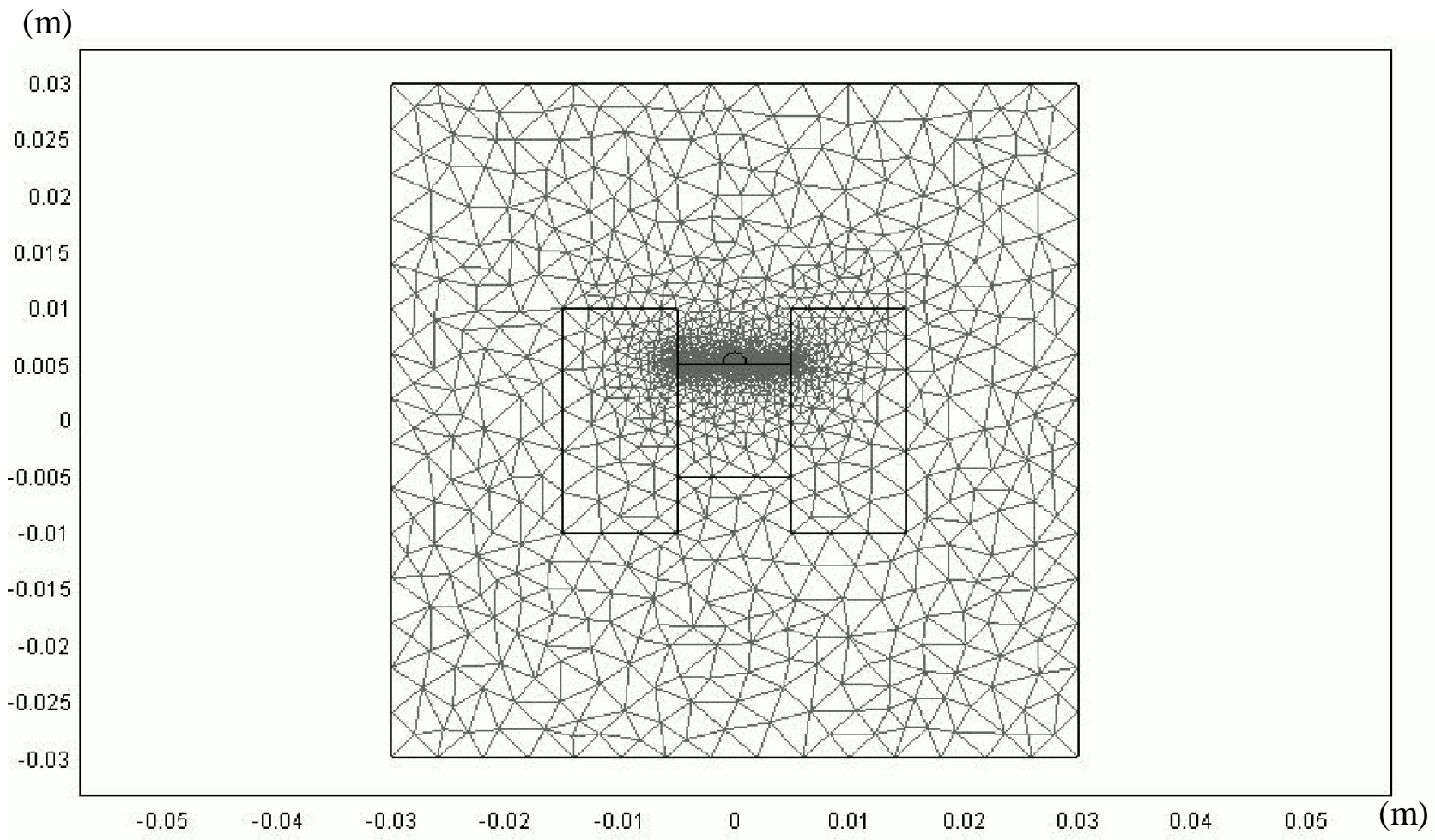

Figure 2: The meshed drawing of domain of study

\section{Results And Discussion:}

The results of these simulations are described using the behavior of electric field and electric potential distributions in the three domains of: air, insulator and water droplet. From those three, the water droplet varies in its radius keeping the contact angle at 90 degrees and also the pollution layer varies in its relative permittivity keeping the thickness at $0.1 \mathrm{~mm}$.

\section{a- Electric Field Distribution:}

Before considering the presence of a water droplet, the electric field will be enhanced at the sharp edges due to the accumulation of charges. The high values of electric field are on two edges, near the HV electrode and near the ground electrode[5]. 


\section{i. Variation of the Water Droplet Diameter:}

In the simulation of the electric field distribution, one water droplet was considered with its radius varying from $0.5 \mathrm{~mm}$ up to $4 \mathrm{~mm}$. The centre point of the water droplet was maintained on the insulator surface precisely. In other words, the contact angle was maintained at 90 degree. Figure (3) shows the electric field contours in case of water droplet diameter of $2 \mathrm{~mm}$ and semi-wetted pollution layer. It is clear that the electric field is enhanced at the triple junction (water droplet, air and polymer).

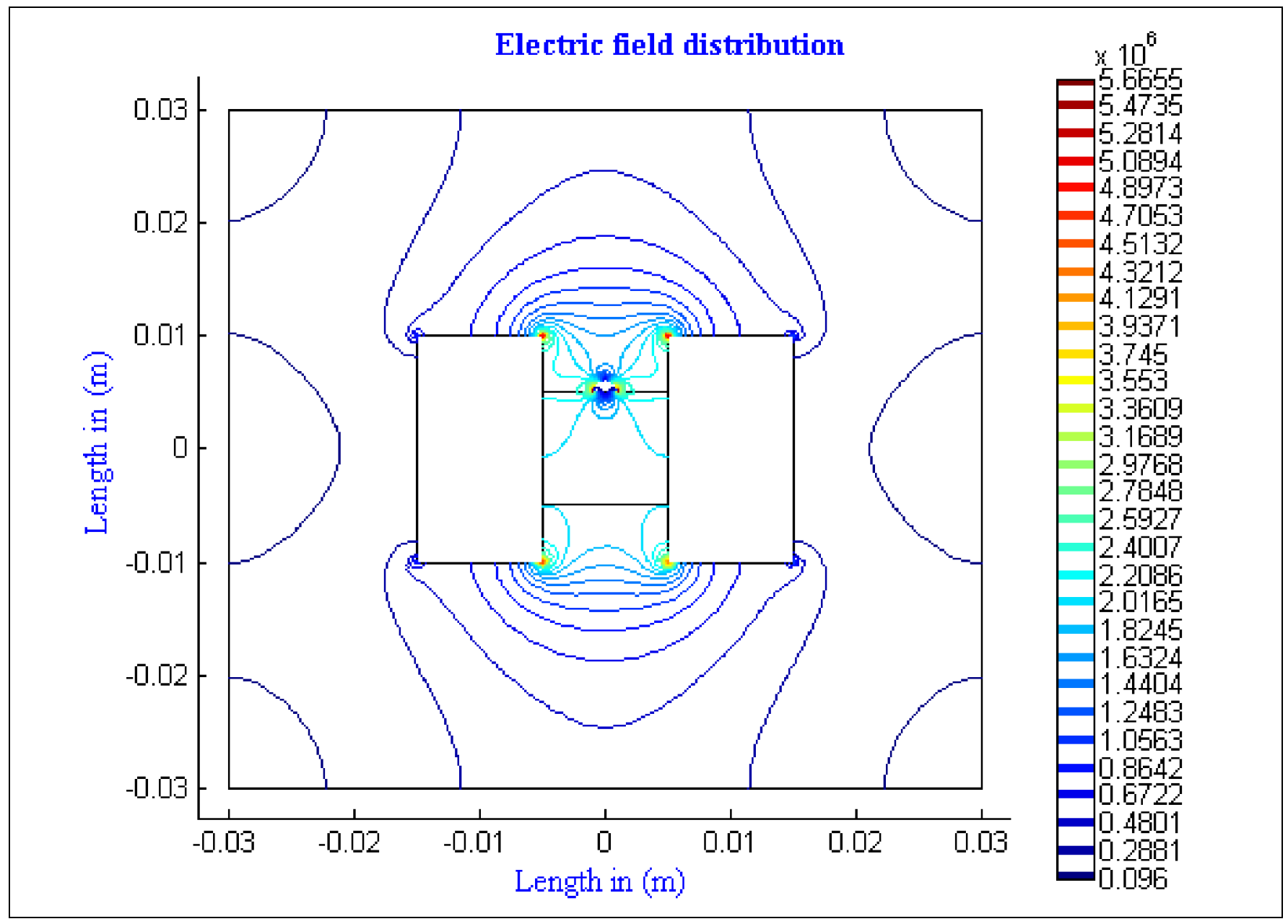

Figure 3: Electric field contours in the case of water droplet $(2 \mathrm{~mm})$ 


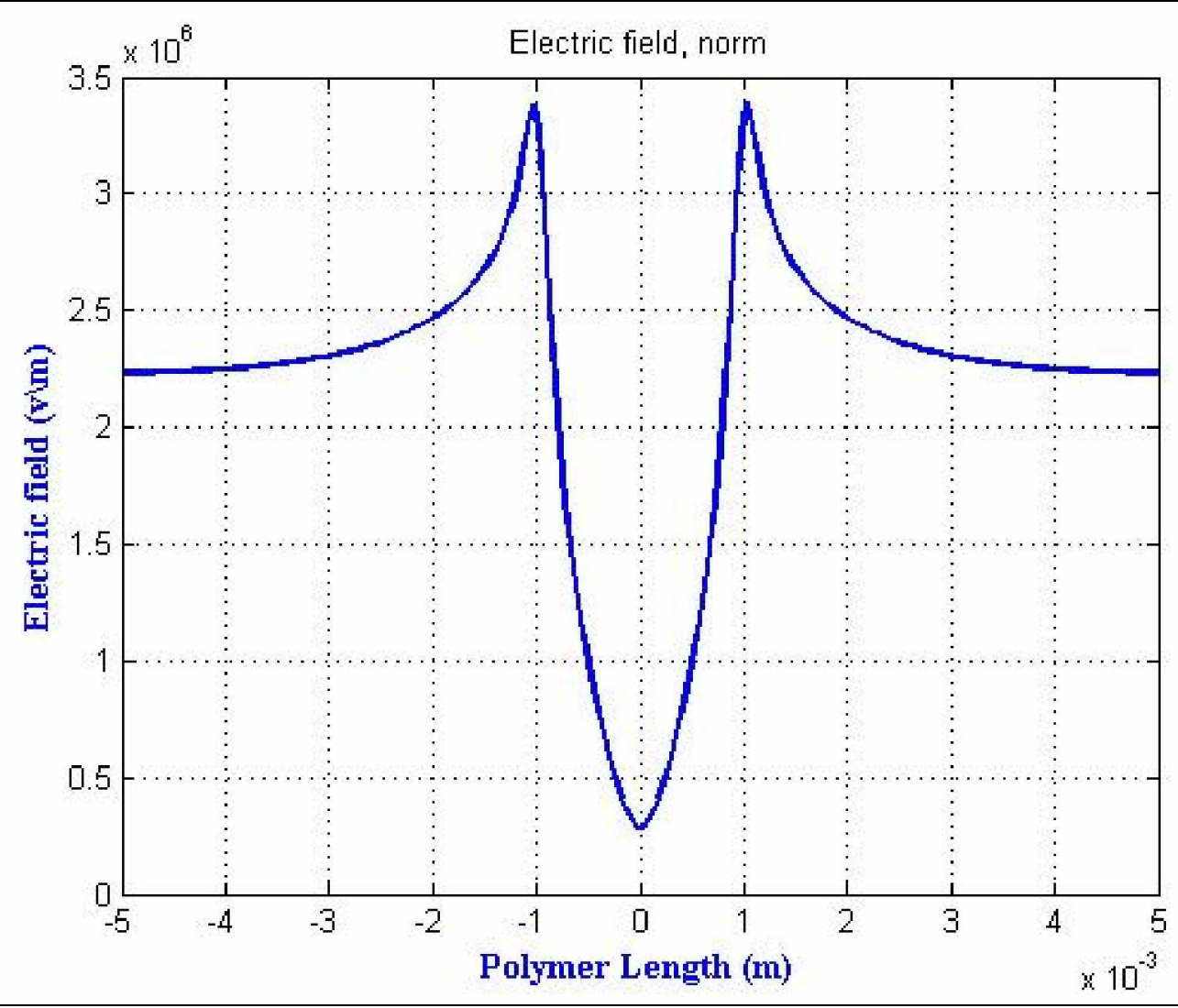

Figure 4: Electric field distribution over the polymer in the case of water droplet $(2 \mathrm{~mm})$

Figure (4) shows the electric field distribution along the polymer length, also it is clear as in figure (3) that the electric field is enhanced at the triple junction. This is because when the water droplet is exposed to the electric field the negative hydrogen $\mathrm{H}^{-}$ions are directed towards the high voltage electrode and the positive oxygen $\mathrm{O}^{+}$ions are directed towards the ground electrode forming two sets of electrode arrangement which redistribute the electric field along the polymer[14].

Figure (5) illustrates the variation of electric field when varying the water droplet diameter. It is obvious that as the water droplet diameter increases the maximum electric field at the triple junction increases. The reason is due to that as the water droplet diameter increases, the water edges become closer to the two electrodes.

The relationship between maximum electric field, $\mathrm{E}_{\max }$, and water droplet diameter, $\mathrm{d}$, can be approximated by the following linear relationship:

$$
E_{\max }=675.5 \times 10^{6} d+E_{c}
$$

Where, $E_{c}$ is the maximum electric field over a clean polymer in (v/m). 


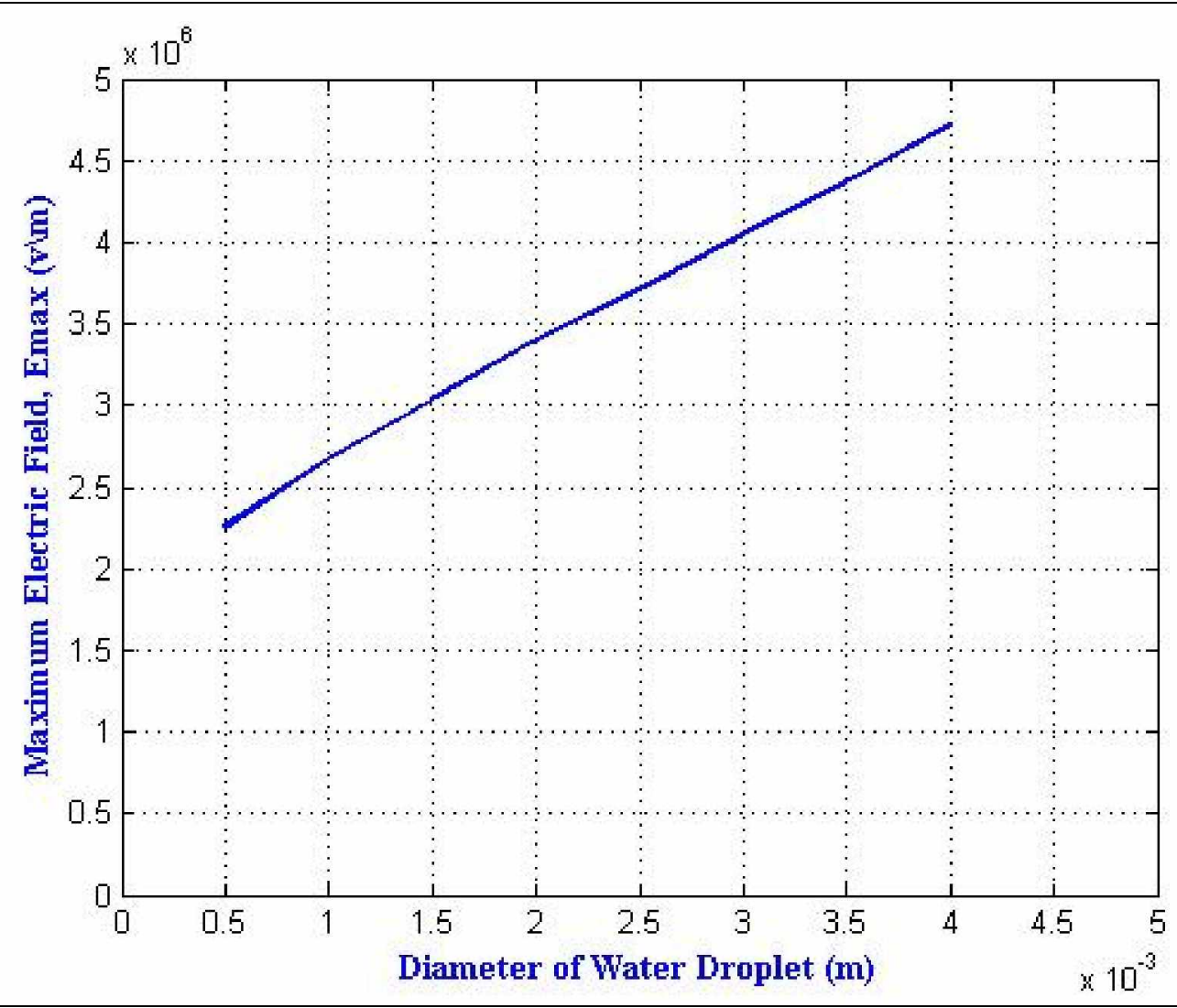

Figure 5: variation of electric field with water droplet diameter

\section{ii. Variation of Pollution Layer Permittivity:}

In the simulation of the electric field distribution, one water droplet was considered; its diameter is kept constant at $2 \mathrm{~mm}$ and contact angle 90 degrees. The pollution layer thickness is $0.1 \mathrm{~mm}$ and its relative permittivity is varied from 2.35 (clean surface) to 80 (fully wetted surface). Values in between represent a semi-wetted surface.

Figure (6) shows the variation of the maximum electric field at the triple junction when varying the relative permittivity of the pollution layer. It is clear that as the relative permittivity of pollution layer increases simulating the wetting condition of the pollution layer, the maximum electric field at the triple junction decreases.

Figure (7) shows the variation of electric field distribution over the polymer surface with varying the relative permittivity of the pollution layer according to three different values: 2.35 (clean polymer), 40 (semi-wetted polymer) and 80 (fully-wetted polymer). 


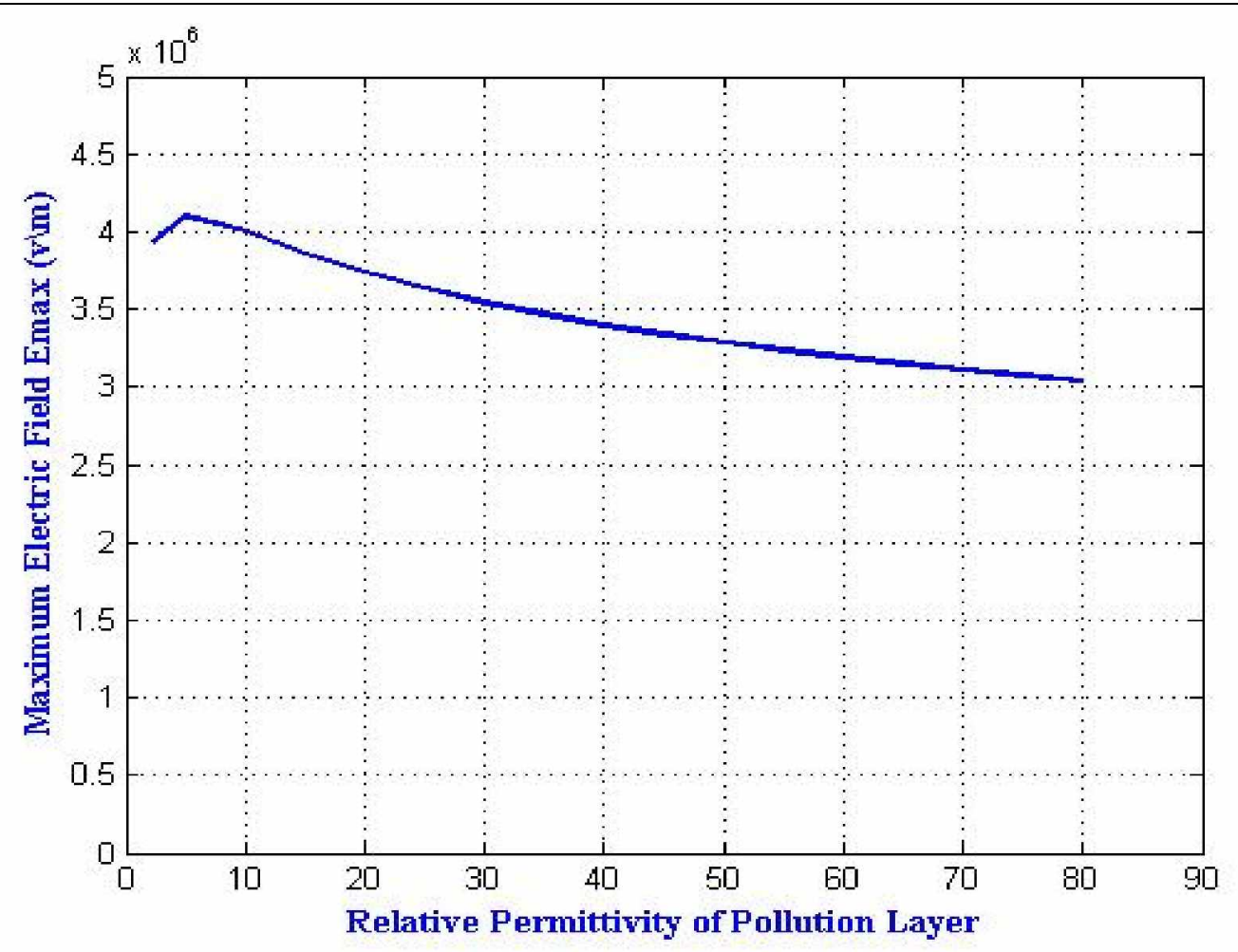

Figure 6: variation of electric field with pollution layer permittivity

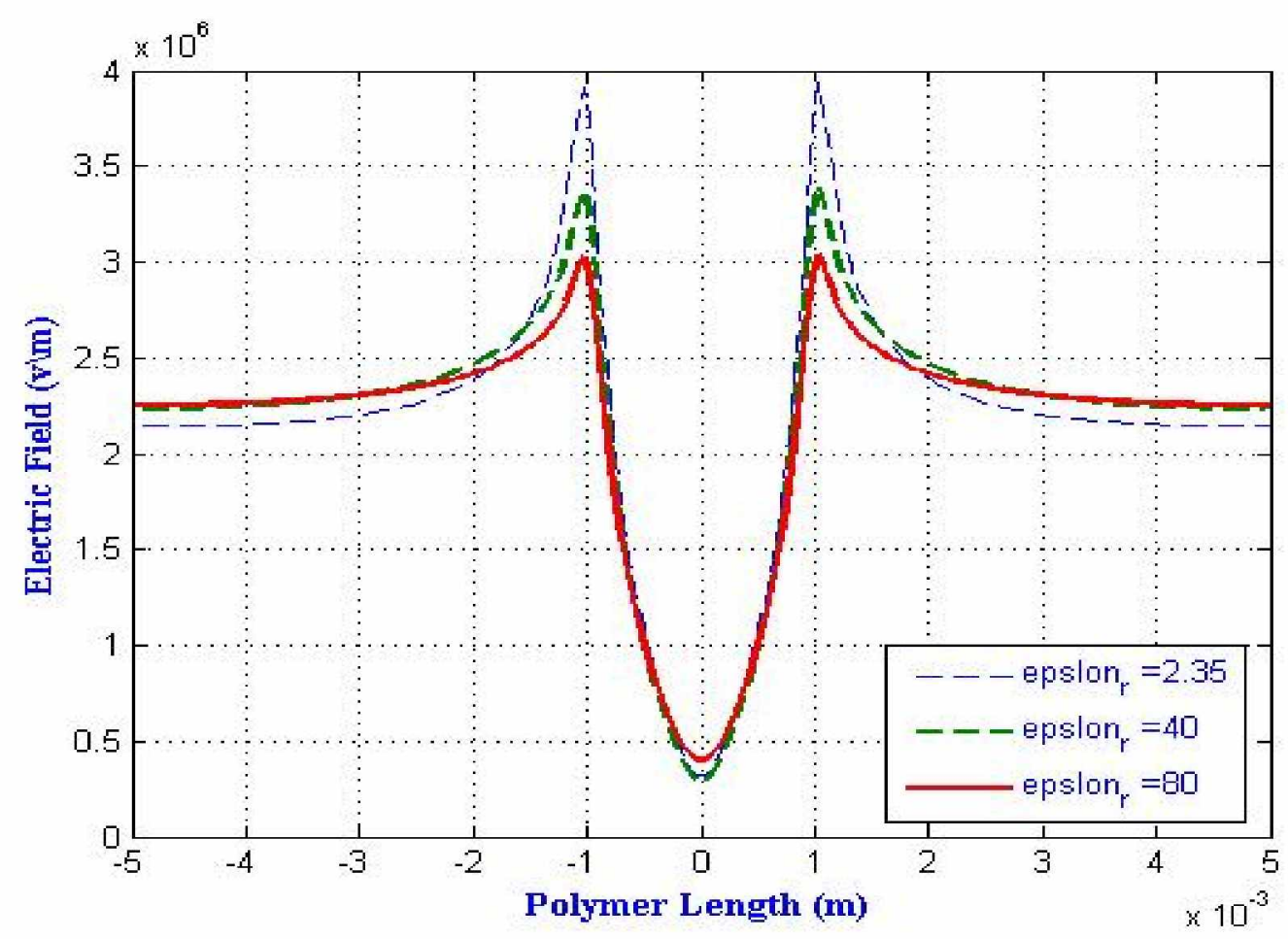

Figure 7: variation of electric field distribution over the polymer surface With varying pollution layer permittivity 


\section{b- Electric Potential Distributions:}

\section{Variation of Water Droplet Diameter:}

Figure (8) shows the potential contour lines over the domain of study, with water droplet diameter (d) of $2 \mathrm{~mm}$, and semi-wetted pollution layer of thickness $0.1 \mathrm{~mm}$. It is clear that the potential contours tend to avoid the water droplet and accumulate at the droplet edges. This will increase the electric field at the water droplet edges i.e. the electric field will be enhanced at triple junction as shown earlier.

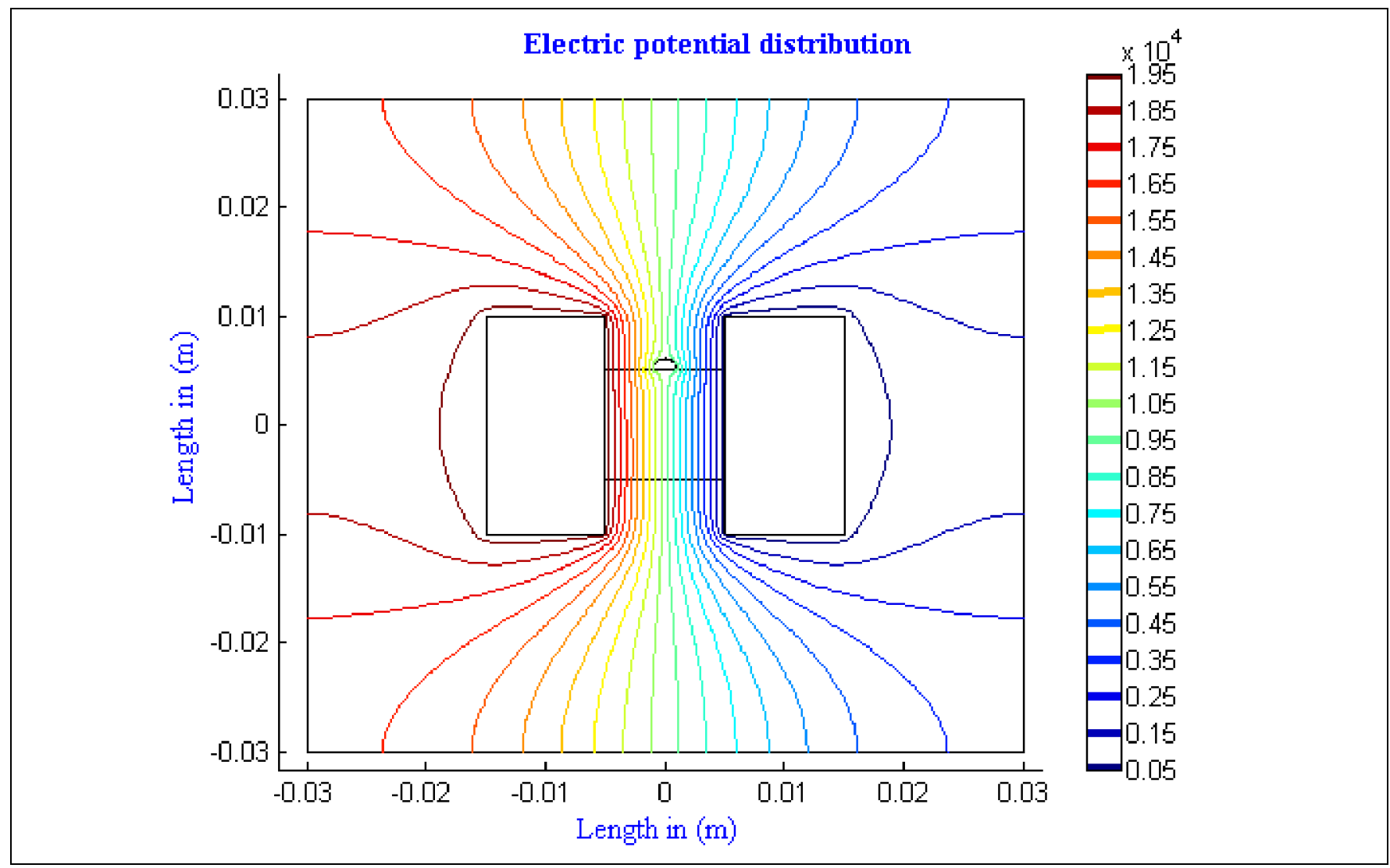

Figure 8: Electric Potential Contours in case of water droplet diameter, $2 \mathrm{~mm}$

Figure (9) shows the electric potential distribution along the polymer surface for two cases of water droplet diameter of $2 \mathrm{~mm}$ and $4 \mathrm{~mm}$. In the two cases, the pollution layer thickness is kept constant at $0.1 \mathrm{~mm}$ and relative permittivity of 40 (semi-wetted pollution layer).

It is clear from the figure that the slope of the curve (electric field $(\mathrm{v} / \mathrm{m})$ ) before and after the water droplet is greater than that through the water droplet region. This illustrates the increasing of the electric field at the water droplet edges (at the triple junction) and decreasing more through the water droplet region. 
Also it is clear that as the water droplet diameter increases the slope increases and hence the electric field increases at the water droplet edges.

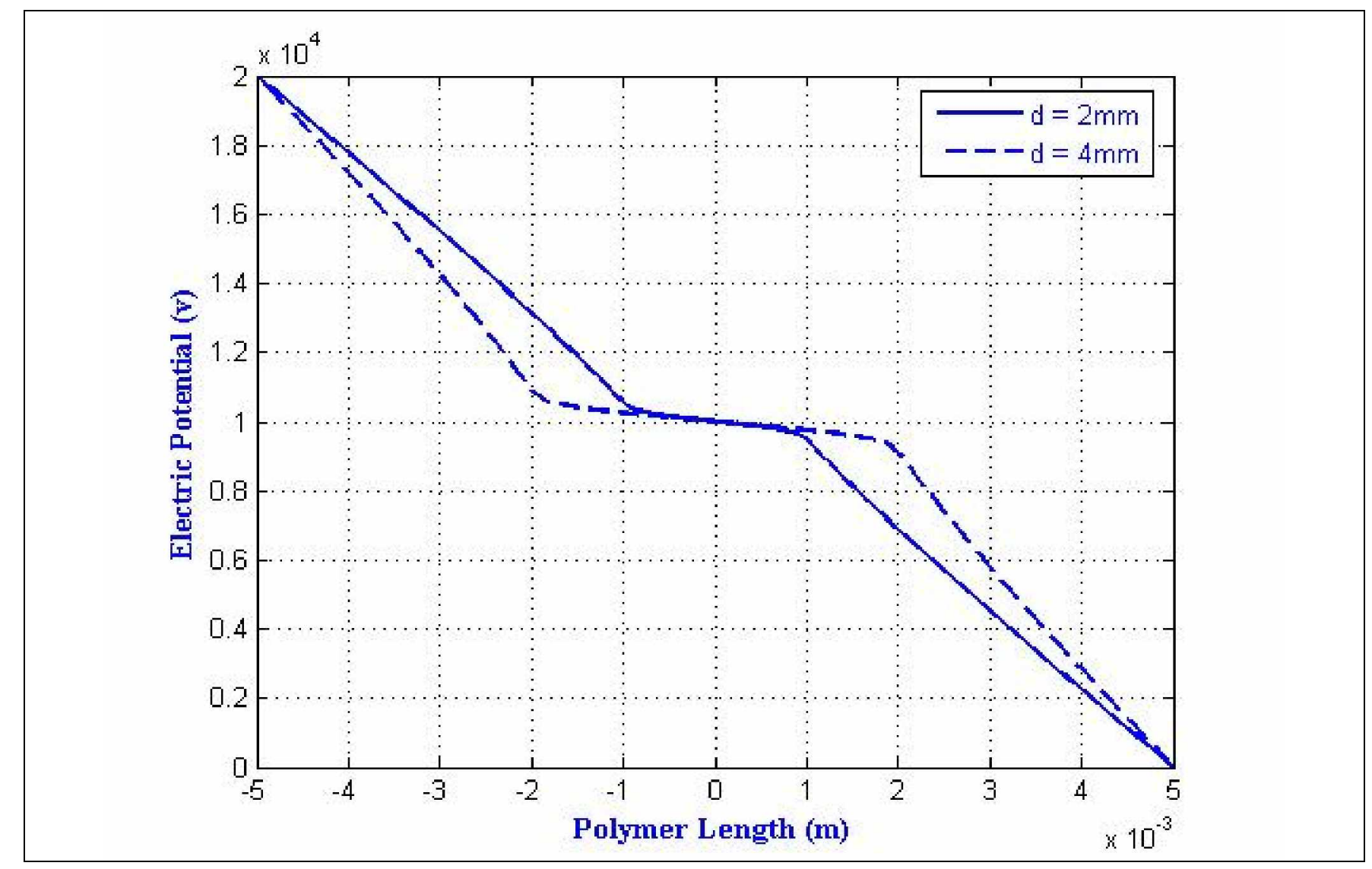

Figure 9: Electric Potential distribution along the polymer surface in case of water droplet diameter, $2 \mathrm{~mm}$ and $4 \mathrm{~mm}$

\section{Conclusions:}

The simulation of electric field and electric potential distributions due to presence of a water droplet on an insulator surface has been carried out based on the finite element method. A sufficiently large number of triangular elements were used to produce accurate results for both the electric field and potential distributions. The maximum electric field around the water droplet will be enhanced as the water droplet diameter increases. An approximate linear relationship was developed to predict the maximum electric field over the polymer surface for any water droplet diameter.

On the other hand, the maximum electric field around the water droplet will decrease as the relative permittivity of the pollution layer increases. The simulation was implemented for three cases (clean polymer, semi-wetted and fully-wetted). Study of the three cases showed that as the water droplet diameter increases, the potential contour plot will be concentrated at their edges, consequently, resulting in an increase in the electric field at the water droplet edges. 


\section{References:}

[1] R. Hackam, "Outdoor HV Composite Polymeric Insulator," IEEE Trans. Dielectr. Electr. Insul, vol. 6, pp. $557: 585,1999$.

[2] I. J. S. Lopes, S. H. Jayaram, and E. A. Chernet, "A Study of Partial Discharges from Water Droplets on a Silicone Rubber Insulating Surface," IEEE Trans. Dielectr. Electr. Insul., vol. 8, pp. 262-268, 2001.

[3] A. J. Phillips, D. J. Childs, and H. M. Schneider, "Aging of Non-Ceramic Insulators Due to Corona from Water Drops," IEEE Trans. Power Del., vol. 14, pp. 1081-1089, 1999.

[4] Y. Zhu, M. Otsubo, C. Honda, Y. Hashimoto, and A. Ohno, "Mechanism for Change in Leakage Current Waveform on a Wet Silicone Rubber Surface-A Study using a Dynamic 3-D Model," IEEE Transactions on Dielectrics and Electrical Insulation, vol. 12, pp. 556 : 565, 2005.

[5] Waluyo, P. M. Pakpahan, and Suwarno, "Influences of water droplet size and contact angle on the electric field and potential distributions on an insulator surface," IEEE 8th Intern. Conf. Properties and Applications of Dielectric Materials, pp. 889 - 892, 2006.

[6] B. N. Pinnangudi, R. S. Gorur, and A. J. Kroese, "Energy Quantification of Corona Discharge on Polymer Insulators," IEEE Conf. Electr. Insul. Dielectr. Phenomena (CEIDP). pp. 315 -318, 2002.

[7] N. M. Ijumba, A. C. Britten, and J. Swartz, "Effect of Water Droplet on Silicone Rubber Insulators under HVDC Potential," 13th Intern. Sympos. High Voltage Eng., Netherlands, pp. 197, 2003.

[8] T. Braunsberger, A. Dziubek, W. Kodoll, U. Schumann, and M. Kurrat, "PD Between Water Droplets Influencing Hydrophobic Processes on SI and EP-resin Systems," 13th Intern. Sympos. High Voltage Eng., Netherlands, pp. 209, 2003.

[9] Z. Cheng, X. Liang, Y. Zhou, S. Wang, and Z. Guan, "Study of Water Droplet Discharge by Electric Field Computation and High-Speed Video," IEEE 7th Intern. Conf. Properties and Applications of Dielectric Materials, vol. 2, pp. 820-823, 2003.

[10] "Femlab 3.1," COMSOL AB, 2004.

[11] YaDong, F., Xishan, W., Fiyu, and Z., "Simulation of Electric Field Distribution of Composite Insulator," Proceedings of The XIV $V^{\text {th }}$ International Symposium on High Voltage Engineering, Tsinghua University, Beijing, China, August 25-29, 2005.

[12] M. N. O. Sadiku, "'Elements of Electromagnetics'," Second ed. Orlando: Saunders College Publishing, 1994., pp. 734,783,

[13] C. S. Desai and J. F. Abel, "Introduction to the Finite Element Method," in A Numerical Approach for Engineering Analysis. New York: Van Nostrand Reinhold, 1972.

[14] K. Katada, Y. Takada, M. Takano, T. Nakanishi, Y. Hayashi, and R. Matsuoka, "Corona Discharge Charactersitics of Water Droplets on Hydrophobic Polymer Insulator Surface," IEEE 6th Intern. Conf. Properties and Applications of Dielectric Materials, vol. 12, pp. 781-784, 2000. 